Artigo Original

Original Article

Ana Clara Sotero Alves ${ }^{1}$

Ana Cristina Côrtes Gama²

Max de Castro Magalhães ${ }^{2}$

Patrícia de Freitas Lopes Genilhú1

Rafaella Cristina Oliveira ${ }^{2}$

Descritores

Voz

Laringe

Qualidade da voz

Disfonia

Acústica da fala

Keywords

Voice

Larynx

Voice quality

Dysphonia

Speech acoustics

Endereço para correspondência:

Rafaella Cristina Oliveira

Rua Benjamim Brandão, 60 Paraíso.

Belo Horizonte (MG) - Brasil

CEP: 30.270-160.

Telefone: (31) 99227-3147

E-mail: rafaellacris_bh@hotmail.com

Recebido em: Fevereiro 10, 2019.

\section{Análise das medidas eletroglotográficas da voz: padrões de normalidade para cantores por meio do programa CSL da Kay Pentax $\mathbb{R}$}

\author{
Electroglotographic voice measurement analysis: \\ normality standards for singers through \\ Kay Pentax® CSL Program
}

\begin{abstract}
Purpose: Analyze the measures of the fundamental frequency, electroglotographic jitter, contact quotient and periodicity of the electroglotographic waves of the singers in habitual voice. Method: A cross-sectional observational study, which selected individuals from singing schools in the central region of the municipality of Belo Horizonte. The sample was non-probabilistic, for convenience consisted of 60 singers aged between 18 and 55 years, 30 males and 30 females. For the electroglotographic record collection, the Kay Pentax TM CSL program, model 6103, the Electroglotographic module was used. The participants were placed in a seated position, and after cleaning the skin of the neck with $70 \%$ alcohol, two electrodes were placed on the wings of the thyroid cartilage at the level of the vocal folds. Subsequently, the participants were instructed to emit the vowel/a/sustained in a habitual way. The measurements of the fundamental frequency $\left(f_{0}\right)$, contact quotient (QC), electroglotographic jitter and periodicity of the electroglotographic wave were analyzed. The data were statistically analyzed using Minitab 17 . Results: The parameters analyzed were statistically significant. The fundamental frequency $\left(f_{0}\right)$ was higher in females $(226.91 \mathrm{~Hz})$ when compared to males $(166.54 \mathrm{~Hz})$. The measure of contact quotient and periodicity was also higher in women ( $40.46 \%$ and $15.51 \%$ respectively) when compared to men (27.45\% and 7.82 , respectively). The jitter parameter was higher in men when compared to women. Conclusion: Women presented higher values in the fundamental frequency $\left(f_{0}\right)$, contact quotient (QC) and periodicity of the wave compared to men. The men presented the jitter value higher than that of the women.
\end{abstract}

Trabalho realizado no Departamento de Fonoaudiologia, Faculdade de Medicina, Universidade Federal de Minas Gerais -UFMG- Belo Horizonte (MG), Brasil.

Departamento de Fonoaudiologia da Universidade Federal de Minas Gerais - UFMG - Belo Horizonte (MG), Brasil.

2 Departamento de Engenharia da Universidade Federal de Minas Gerais - UFMG - Belo Horizonte (MG), Brasil.

Conflito de interesses: Nada a declarar.

Fonte de financiamento: Fapemig (APQ-02594-15) 


\section{INTRODUÇÃO}

A voz é produzida no trato vocal e é resultado da integração entre a fonação, ou seja, entre a vibração das pregas vocais e a ressonância. Para a produção vocal ocorrer sem esforço, é necessária perfeita harmonia muscular, porém fatores, como a influência do meio em que se vive e a cultura a que se pertence, fazem com que não existam padrões e nem limites definidos sobre a classificação da voz normal ${ }^{(1)}$.

Os indivíduos que utilizam a voz como instrumento de trabalho são considerados profissionais da voz. Dentre estes, os cantores, também denominados de "atletas da voz", representam uma classe profissional geralmente de alta demanda vocal, com diferentes graus de exigências e requintes, sendo necessários alguns ajustes específicos, como interação coordenada entre respiração, articulação e ressonância ${ }^{(2)}$. A avaliação vocal destes profissionais é de fundamental importância, sendo eficaz no diagnóstico e tratamento de uma alteração vocal, e para acompanhar o progresso do tratamento instituído.

O estudo da vozé multidimensional e é indispensável recorrer às análises múltiplas, como as análises perceptivo-auditiva e acústica da voz, e as avaliações aerodinâmica e eletroglotográfica, que permitem um conhecimento amplo, adequado e eficaz da função laríngea e da qualidade vocal ${ }^{(3)}$.

Atualmente, a avaliação fonoaudiológica da voz pode ser feita por meio da avaliação perceptivo-auditiva, a qual, apesar de ser subjetiva e depender do julgamento de avaliadores, é considerada padrão ouro na clínica vocal, ou pela análise acústica, uma abordagem objetiva, que utiliza um programa de computador que demonstra medidas ou gráficos realizados a partir dos sinais de voz capturados ${ }^{(4)}$. A análise acústica surgiu como forma de tornar a avaliação vocal mais objetiva, além de permitir maior precisão diagnóstica, identificar e documentar os resultados do tratamento em curto e longo prazo ${ }^{(5)}$.

A eletroglotografia (EGG) também é uma técnica objetiva, não invasiva e de simples medida, empregada para estimar a movimentação das pregas vocais durante a fonação ${ }^{(6)}$. Esta técnica foi criada por Fabre (1957) ${ }^{(7)}$, na qual um par de eletrodos de placa é fixado externamente no pescoço em ambos os lados da cartilagem tireóidea. Durante a fonação, um sinal de alta frequência com corrente elétrica muito baixa (aproximadamente entre $300 \mathrm{KHz}$ e $5 \mathrm{MHz}$ ) é emitido por um eletrodo e detectado pelo outro contralateral. A partir do momento em que as pregas vocais vibram, a corrente elétrica é modulada em amplitude, que é detectada pelo dispositivo, gerando um sinal (onda EGG) que reflete a mudança na impedância elétrica na laringe ${ }^{(8)}$. Esse exame parte do princípio de que o tecido laríngeo é um ótimo condutor elétrico e que o ar apresenta pouca condutância ${ }^{(6,8)}$. Assim, no caso da vibração glótica, quanto mais fechadas as pregas vocais, maior é a energia elétrica que passa entre elas e menor é a impedância ${ }^{(9)}$.

As principais vantagens da eletroglotografia estão relacionadas ao fato de ser uma técnica não invasiva, que não interfere com o processo de fala e cuja utilização não está condicionada ao ruído ambiental. Em comparação com outras técnicas, é a que melhor representa a fase de fechamento das pregas vocais e o cálculo da frequência fundamental $\left(f_{0}\right)$. Esta técnica permite ainda apresentar apenas os efeitos da fonte glótica sem interferência do filtro (trato vocal) ${ }^{(10)}$. Portanto, a EGG fornece uma visão sobre o comportamento das pregas vocais que não é obtida por nenhum outro meio prático e pode desempenhar um papel importante na avaliação clínica, terapêutica e no monitoramento de cantores, bem como promover nosso entendimento básico do funcionamento da laringe ${ }^{(9)}$.

Estabelecer padrões de normalidade das avaliações vocais é de fundamental importância para atuação com os profissionais da voz. A padronização educa, simplifica e economiza tempo, dinheiro e esforço, além de garantir certificação ${ }^{(11)}$. As pesquisas de padronização de medidas podem sofrer diferenciação devido aos programas que analisam a voz e a fala, ao modo de calcular os parâmetros acústicos, além do local da gravação, do ruído ambiental e do gênero e da idade do falante ${ }^{(12)}$.

Não encontramos na literatura nacional estudos utilizando medidas eletroglotográficas extraídas por meio do programa CSL da Kay Pentax ${ }^{\mathrm{TM}}$ em cantores. Dessa forma, o objetivo deste estudo foi analisar as medidas de frequência fundamental $\left(f_{0}\right)$, a medida do quociente de contato (QC), o jitter eletroglotográfico e a periodicidade das ondas eletroglotográficas, para que possamos obter dados de normalidade para cantores em registro modal e comparar os parâmetros eletroglotográficos em ambos os sexos.

\section{MÉTODO}

Estudo observacional de corte transversal que selecionou indivíduos de escolas de canto da região central do município de Belo Horizonte. A amostra foi não probabilística, por conveniência, composta por 60 indivíduos cantores com faixa etária entre 18 e 55 anos, sendo 30 homens com idade de 19 a 48 anos (média de 28,6 anos) e 30 mulheres com idade de 18 a 36 anos (média de 26,1 anos), no período de novembro de 2015 a janeiro de 2016. Os grupos foram pareados por idade $(\mathrm{p}=0,21)$.

Como critério de inclusão, os participantes foram submetidos à análise perceptivo-auditiva realizada por uma fonoaudióloga especialista em voz e ao exame de videolaringoscopia de alta velocidade realizado por um otorrinolaringologista, a fim de certificar que não possuíam alterações vocais e laríngeas. Ambos foram realizados no Observatório de Saúde Funcional em Fonoaudiologia da Faculdade de Medicina da Universidade Federal de Minas Gerais (OSF/UFMG). Foram excluídos indivíduos fumantes e mulheres grávidas ou no período menstrual.

Todos os participantes foram informados sobre objetivo e procedimentos do estudo, e após a concordância, assinaram o Termo de Consentimento Livre e Esclarecido (TCLE). Este trabalho foi aprovado pelo Comitê de Ética em Pesquisa (COEP), da Universidade Federal de Minas Gerais, sob o número CAAE 48085815.2.0000.5149.

Para a coleta do registro eletroglotográfico das vozes, foi utilizado o programa CSL da Kay Pentax ${ }^{\mathrm{TM}}$, model 6103, módulo Electroglottography, instalado em um computador da marca Dell ${ }^{\circledR}$, modelo Optiplex GX260, com placa de som profissional

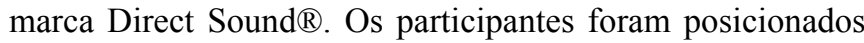
sentados, confortavelmente, e, após a higienização da pele do pescoço com álcool a $70 \%$, dois eletrodos foram colocados nas alas da cartilagem tireóidea no nível das pregas vocais (Figura 1). 
Posteriormente, os participantes foram orientados a emitir a vogal /a/ sustentada de forma habitual, no registro modal.
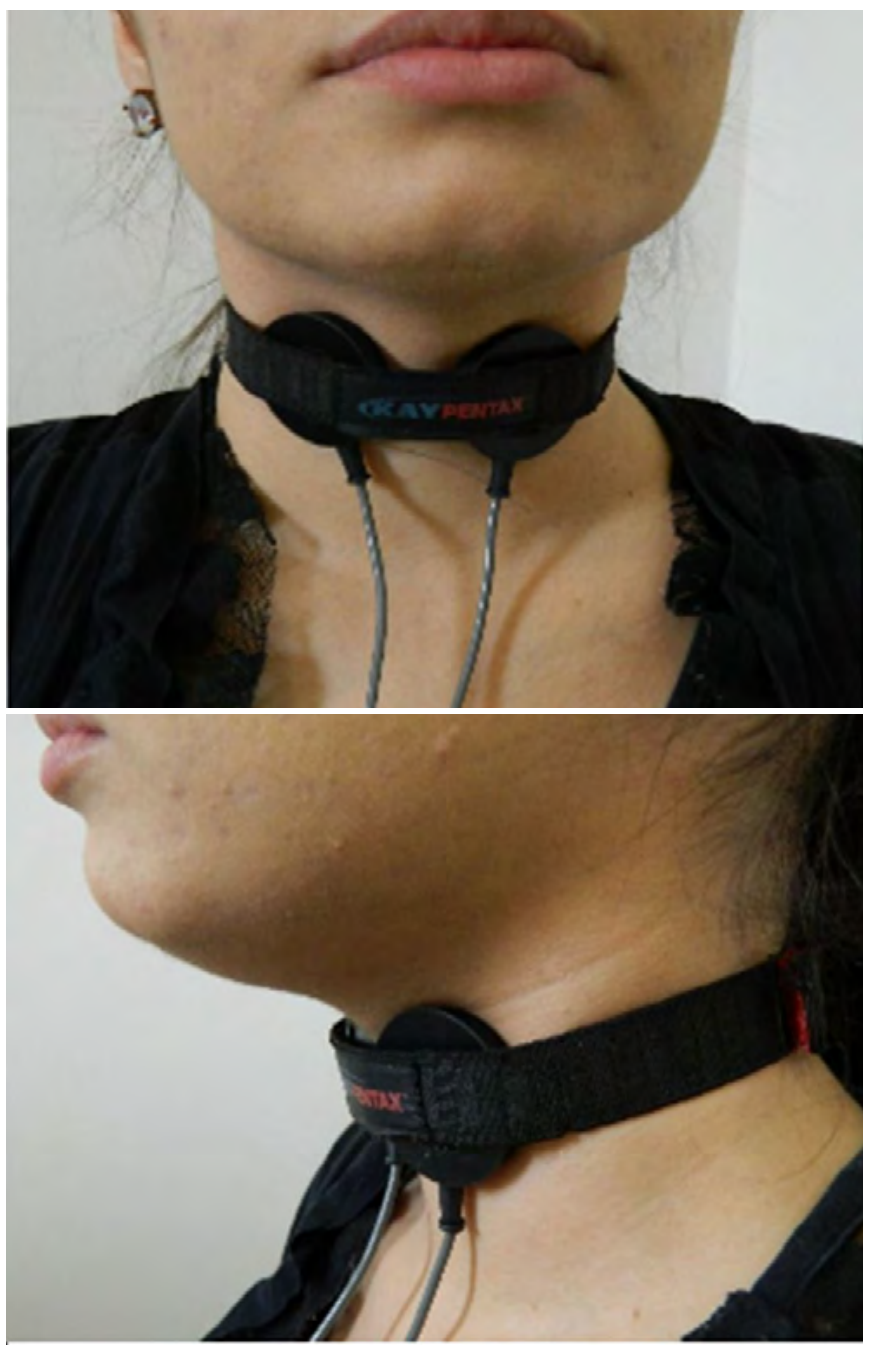

Figura 1. Posição dos eletrodos afixados na nas alas da cartilagem tireóidea no nível das pregas vocais para avaliação eletroglotográfica

Fonte: Tirada pelas autoras

Nesta análise, o padrão de contato das pregas vocais pode ser afetado por fatores, como: a contração da musculatura perilaríngea, do pescoço e da fáscia superficial, levando a alterações na transmissão e captação no sinal elétrico recolhido; o movimento das outras estruturas do trato vocal, e a existência de excesso de muco na cavidade glótica. Outra possível alteração, relacionada com os achados na onda eletroglotografia, é o suor $\left.^{(10}\right)$. Assim, os indivíduos foram orientados a não deglutir e não movimentar o pescoço durante a gravação. Além disso, por se tratar de uma população de cantores que possui maior consciência corporal e treinamento vocal, a presença destes artefatos foram minimizados.

Foram analisadas as medidas eletroglotográficas de:

- frequência fundamental $\left(f_{0}\right)$, que corresponde ao número de ondas sonoras produzidas em um intervalo de tempo de um segundo. A unidade de medida é o hertz $(\mathrm{Hz})$.

- quociente de contato $(\mathrm{QC})$, que corresponde à medida de contato das pregas vocais durante a fonação. É expresso em porcentagem $(\%)$.

- jitter eletroglotográfico, que corresponde às mudanças de frequência entre os ciclos fonatórios nos sinais de EGG. A unidade de medida é o hertz $(\mathrm{Hz})$.

- periodicidade da onda eletroglotográfica, cujas ondas devem apresentar os mesmos padrões em sua forma. É expresso em porcentagem (\%).

Para análise estatística dos dados, foi utilizado o programa Minitab 17. Primeiramente, realizou-se uma análise descritiva dos parâmetros eletroglotográficos com valores mínimos, máximos, média e desvio-padrão. Foram comparados os grupos de homens e mulheres, utilizando o teste estatístico não paramétrico MannWhitney, com nível de significância de $5 \%$. Posteriormente, foi calculado, para cada parâmetro analisado, o Poder do Teste, utilizando o programa estatístico G.Power 3.1®.

\section{RESULTADOS}

A tabela a seguir (Tabela 1) mostra os valores de mínimo, máximo e desvio padrão para os parâmetros eletroglotográficos de homens e mulheres cantores.

Todos os parâmetros analisados foram estatisticamente significantes. As mulheres apresentaram valores maiores na frequência fundamental $\left(f_{0}\right)$, no quociente de contato (QC) e na periodicidade, em comparação aos valores verificados nos homens. Os homens apresentaram o valor de jitter maior que o das mulheres.

O Poder do Teste se mostrou adequado para todas as variáveis analisadas.

Tabela 1.Parâmetros eletroglotográficos em homens e mulheres cantores

\begin{tabular}{|c|c|c|c|c|c|c|c|c|c|c|}
\hline \multirow{2}{*}{$\begin{array}{l}\text { Parâmetros } \\
\text { eletroglotográficos }\end{array}$} & \multicolumn{4}{|c|}{ Homem } & \multicolumn{4}{|c|}{ Mulher } & \multirow{2}{*}{$\mathbf{P}$} & \multirow[b]{2}{*}{$\begin{array}{l}\text { Poder do } \\
\text { Teste }\end{array}$} \\
\hline & Mínimo & Máximo & Média & $\begin{array}{l}\text { Desvio } \\
\text { Padrão }\end{array}$ & Mínimo & Máximo & Média & $\begin{array}{l}\text { Desvio } \\
\text { Padrão }\end{array}$ & & \\
\hline $\begin{array}{l}\text { Frequência } \\
\text { Fundamental }\left(\mathrm{F}_{0}\right)\end{array}$ & 96,80 & 255,79 & 166,54 & 43,51 & 117,64 & 362,73 & 226,91 & 41,59 & $0,0000^{*}$ & $99,9 \%$ \\
\hline $\begin{array}{l}\text { Quociente de contato } \\
\text { (QC) }\end{array}$ & 3,340 & 63,040 & 27,455 & 13,776 & 11,840 & 52,390 & 40,460 & 10,923 & $0,0002^{*}$ & $99,5 \%$ \\
\hline Jitter & 0,850 & 25,490 & 11,016 & 7,118 & 0,3800 & 34,4200 & 4,7860 & 6,2445 & $0,0001^{*}$ & $99,1 \%$ \\
\hline Periodicidade & $-0,3400$ & 34,4800 & 7,8247 & 9,4712 & $-0,570$ & 42,170 & 15,513 & 9,296 & $0,0027^{\star}$ & $95,3 \%$ \\
\hline
\end{tabular}

Legenda: * Diferença estatisticamente significante. Teste estatístico: Mann-Whitney.

Fonte: Elaboração própria a partir de dados coletados. 


\section{DISCUSSÃO}

A eletroglotografia é uma avaliação que oferece a possibilidade de estudo do que se refere ao contato das pregas vocais durante o ciclo glótico, sendo utilizada para avaliar a função vocal ${ }^{(7,11,12)}$. É um instrumento antigo que apresenta várias interpretações em relação às medidas da onda e seus parâmetros qualitativos ${ }^{(6)}$. Os parâmetros eletroglotográficos podem sofrer alteração devido à diferença dos programas e aos critérios de avaliação ${ }^{(10)}$. Portanto, enquanto os valores normais não tiverem sido validados, a aplicação clínica da EGG será limitada.

A padronização tem como objetivo fornecer dados normativos sobre a compatibilidade, a segurança e a variabilidade das medições entre pessoas. No caso dos procedimentos que avaliam a voz, a padronização tem sido enfatizada em diversas pesquisas ${ }^{(14,15)}$.

Várias medidas objetivas podem ser obtidas com a análise do traçado eletroglotográfico. Podemos analisar a frequência fundamental da vibração, as medidas de perturbação da amplitude e do shimmer, de perturbação da frequência e do jitter, e o quociente de fechamento das pregas vocais. Em relação à extração dos valores da frequência fundamental, a eletroglotografia é um método aceito para medir tal parâmetro ${ }^{(14)}$. Segundo a literatura, a frequência fundamental na EGG é de mais fácil extração do que na análise acústica da onda sonora, por representar ciclos mais nítidos ${ }^{(1)}$. Neste estudo, observou-se uma diferença estatisticamente significante em relação aos sexos. Tais achados corroboram com a literatura ${ }^{(11,14)}$, que também descreve valores estatisticamente significantes para média de $f_{0}$ entre homens e mulheres. Porém, discordam de estudo de Guimarães e Abberton ${ }^{(9)}$, que não encontrou diferença estatisticamente significante para esse parâmetro eletroglotográfico.

A média da frequência fundamental encontrada no presente estudo, para voz em registro modal de homens $(166,54 \mathrm{~Hz})$, foi superior à encontrada por Faria et al. ${ }^{(14)}$, que foi de $127,77 \mathrm{~Hz}$, e a encontrada por Ma et al. ${ }^{(11)}$, que foi de $122,28 \mathrm{~Hz}$. A média do mesmo parâmetro encontrada para as mulheres neste estudo foi de $226,91 \mathrm{~Hz}$, também se mostrando superior às encontradas por Faria et al. ${ }^{(14)}(204,87 \mathrm{~Hz})$ e por Ma et al. ${ }^{(11)}(205,87 \mathrm{~Hz})$. Tais diferenças podem ser explicadas pelo fato de as medidas terem sido obtidas por diferentes programas de extração da eletroglotografia. Entretanto, o estudo com mulheres adultas com nódulos vocais e presença de fenda glótica, e com mulheres sem alterações laríngeas mostrou que a média da frequência fundamental foi de $211,69 \mathrm{~Hz}$ para vozes normais ${ }^{(13)}$, valor que se aproxima deste estudo.

Quanto aos resultados obtidos para o quociente de contato (QC), nota-se que houve diferença estatisticamente significante entre homens e mulheres cantores. Os homens apresentaram valores da média de QC inferiores aos das mulheres, sendo $27,45 \%$ para homens e $40,46 \%$ para mulheres. Tal achado difere do estudo que mostrou valores de quociente de contato maiores para homens, quando comparados aos das mulheres ${ }^{(15)}$.

Outro estudo descrito na literatura mostra que a média do quociente de contato foi de $42,52 \%$ e $45,56 \%$ para grupo estudo e controle, respectivamente, não havendo diferença significante entre grupos ${ }^{(14)}$. Apesar de não haver diferença significante entre os grupos para os achados da média de QC, os valores encontrados aproximam-se dos valores encontrados de QC em mulheres no nosso estudo. Tais achados podem ser justificados por este estudo ter usado o programa CSL modelo 4300 da Kay Elemetrics ${ }^{(14)}$.

No que se refere à extração do jitter eletroglotográfico, notou-se também uma diferença estatisticamente significante entre os sexos. O sexo feminino apresentou um valor inferior $(4,78 \mathrm{~Hz})$, se comparado ao sexo masculino $(11,01 \mathrm{~Hz})$. Os dados encontrados para vozes femininas concordam com estudo com indivíduos sem queixas vocais, no qual os valores de jitter absoluto variaram de 1,33 a $6,36 \mathrm{~Hz}$ para vozes femininas ${ }^{(14)}$. Concordam também com estudo com mulheres adultas com nódulos vocais e fenda glótica, e com mulheres sem alterações laríngeas, que apresentou valor médio de jitter de 1,21 Hz e 2,9 $\mathrm{Hz}$ para o grupo estudo e controle, respectivamente ${ }^{(13)}$.

Entretanto, discordam em relação aos valores encontrados para vozes masculinas $(0,61 \mathrm{a} 4,37 \mathrm{~Hz})^{(14)}$. Tal diferença pode ser justificada pelo fato de as pesquisas utilizarem programas de análise eletroglotográfica distintos e, portanto, diferentes métodos matemáticos para extração da aperiodicidade de frequência em curto prazo da onda eletroglotográfica.

Não foram encontrados estudos relacionados às medidas eletroglotográficas de periodicidade da onda eletroglotográfica correlacionando os sexos. A análise dos parâmetros eletroglotográficos realizada neste estudo, apesar do número reduzido de indivíduos avaliados, poderá contribuir para análise das medidas eletroglotográficas de vozes de cantores extraídas por meio do programa CSL da Kay Pentax ${ }^{\mathrm{TM}}$.

Ressalta-se a necessidade de novos estudos com uma amostra mais significativa que comparem as medidas de frequência fundamental, quociente de contato e periodicidade, confirmando a padronização dessas medidas e, consequentemente, garantindo maior precisão na avaliação vocal.

\section{CONCLUSÃo}

Homens e mulheres cantores com laringe normal apresentaram medidas eletroglotográficas distintas. A frequência fundamental $\left(f_{0}\right)$ foi maior nas mulheres quando comparada com a verificada nos homens, assim como a medida de periodicidade e o quociente de contato. Ao contrário, o parâmetro de jitter foi maior nos homens, quando comparado ao das mulheres.

\section{AGRADECIMENTOS}

À Fundação de Amparo à Pesquisa do Estado de Minas Gerais - Fapemig (APQ-02594-15), pelo apoio com fomento à pesquisa.

\section{REFERÊNCIAS}

1. Behlau M, Azevedo R, Pontes P. Conceito da voz normal e classificação das disfonias. In: BEHLAU, Mara (Org.). Voz - O livro do especialista. v.1. Rio de Janeiro: Revinter, 2004

2. Phyland DJ. The impact of vocal load on the vocal function of professional music theatre singers [PhD thesis]. Australia: Monash Universtity; 2014

Dejonckere PH, Bradley P, Clemente P, Cornut G, Crevier-Buchman L, Friedrich G, Van de Heyning P, et al. A basic protocol for functional 
assessment of voice pathology, especially for investigating the efficacy of (phonosurgical) treatments and evaluating new assessment techniques: guideline elaborated by the Committee on Phoniatrics of the European Laryngological Society (ELS). Eur Arch Otorhinolaryngol. 2001;258:77-82. PMid:11307610. https://doi.org/10.1007/s004050000299.

3. Behlau M, Pontes P. Avaliação e tratamento das disfonias. São Paulo: Lovise; 1995

4. Ambrozic MK, Boltezar IH, Hren NI. Changes of some functional speech disorders after surgical correction of skeletal anterior open bite. International Journal of Rehabilitation Research. 2015;38(3):246-52. PMid:26164798. https://doi.org/10.1097/MRR.0000000000000123.

5. Rothenberg MA. Multichannel Electroglottograph. J Voice. 1992

6. Fabre P. Une perocédé électrique d'inscription de l'accolement glottique au course de la phonation: Glottographie de haute frequence. Bulletin de l'Académie Nationale de Médicine. 1957; 141:66-9 IN: Rothenberg M. A multichannel electroglottograph. J Voice. 1992;6(1):36-43. https://doi. org/10.1016/S0892-1997(05)80007-4

7. Baken RJ. Electroglottography. J Voice. 1992;6(2): 98-110

8. Guimarães, IG, Abberton E. Fundamental Frequency in Speakers of Portuguese for Different Voice Samples. J.Voice. 2005;9(4):592-606. https://doi.org/10.1016/j.jvoice.2004.11.004

9. Felippe ACN, Grillo MHMM, Grechi TH. Standardization of acoustic measures for normal voice patterns. Braz J Otorhinolaryngol. 2006;72(5):659-64. https://doi.org/10.1016/S1808-8694(15)31023-5
10. Ma EPM, Love AL. Eletroglottographic Evaluation of Age and Gender Effects During Sustained Phonation and Connected Speech. J. Voice. 2010;24(2):148. PMid:19481415. https://doi.org/10.1016/j.jvoice.2008.08.004.

11. Herbst CT, Howard D, Schlomicher-Their J, Salzburg. Using Electroglottographic Real-Time Feedback to Control Posterior Glottal Adduction during Phonation. J Voice. January, 2010; 24(1):72-85. PMid:19185453. https:// doi.org/10.1016/j.jvoice.2008.06.003.

12. Mourão AM, Gama ACC, Bassi IB. Avaliação eletroglotográfica de mulheres disfônicas com lesão de massa. Rev. CEFAC [online]. 2011;13(6):1073-80. https://doi.org/10.1590/S1516-18462011005000097

13. Faria BS, Oliveira KV, Silva JPG, Reis C, Ghio A, Gama ACC. Electroglottography of speakers of Brazilian Portuguese through Objective Multiparameter Vocal Assessment (EVA). Braz J Otorhinolaryngol. 2012;78:29-34. https://doi.org/10.1590/S1808-86942012000400007

14. Awan SM, Awan JA. The effect of gender on measures of eletroglottographic contact quotient. J Voice. 2013;27(4):433-40. PMid:23809567. https://doi. org/10.1016/j.jvoice.2013.03.007.

\section{Contribuição dos autores}

ACSA, RCO, PFLG: participaram da idealização do estudo, revisão bibliográfica, coleta, análise e interpretação dos dados coletados e redação do artigo; ACCG, MCM: participaram na condição de orientadores, da idealização do estudo, análise, interpretação dos dados e redação do artigo. 